\title{
Heat kernel transform for nilmanifolds associated to the Heisenberg group
}

\section{Bernhard Krötz, Sundaram Thangavelu and Yuan Xu}

\begin{abstract}
We study the heat kernel transform on a nilmanifold $M$ of the Heisenberg group. We show that the image of $L^{2}(M)$ under this transform is a direct sum of weighted Bergman spaces which are related to twisted Bergman and Hermite-Bergman spaces.
\end{abstract}

\section{Introduction}

Let us consider a complete analytic Riemannian manifold $M$ and let us denote by $k_{t}(x, y)$ the heat kernel on it. We fix $t>0$ and draw our attention to the map

$$
K: M \rightarrow L^{2}(M), \quad m \mapsto k_{t}(m, \cdot) .
$$

This assignment is analytic and hence $K$ admits an analytic extension to a holomorphic map

$$
K^{\sim}: M_{\mathbb{C}} \rightarrow L^{2}(M), \quad z \mapsto k_{t}^{\sim}(z, \cdot)
$$

with $M_{\mathbb{C}}$ a Stein tube surrounding $M$. Consequently, we obtain a map

$$
T_{t}: L^{2}(M) \rightarrow \mathcal{O}\left(M_{\mathbb{C}}\right) ; T_{t}(f)(z)=\int_{M} f(m) k_{t}^{\sim}(z, m) d m
$$

which we call the the heat kernel transform. The basic problem now is to find appropriate tubes $M_{\mathbb{C}}$ and then characterize the image of $T_{t}$. This has been successfully carried out for the following pairs $\left(M, M_{\mathbb{C}}\right)$ :

- $\left(\mathbb{R}^{n}, \mathbb{C}^{n}\right) \quad[2]$.

- $\left(U, U_{\mathbb{C}}\right)$ with $U$ a compact Lie group and $U_{\mathbb{C}}$ its universal complexification [7].

2000 Mathematics Subject Classification: Primary: 22E30. Secondary: 35H20, 35K05, $58 \mathrm{~J} 35$.

Keywords: Heisenberg group, nilmanifolds, Bergman spaces, heat kernel, Hermite and Laguerre functions. 
- $\left(U / K, U_{\mathbb{C}} / K_{\mathbb{C}}\right)$ with $U / K$ a compact symmetric space [11].

- $\left(\mathbb{H}, \mathbb{H}_{\mathbb{C}}\right)$ with $\mathbb{H}$ the Heiseberg group and $\mathbb{H}_{\mathbb{C}}$ its universal complexification [8].

- $(G / K, \Xi)$ with $G / K$ a Riemannian symmetric space of the non-compact type and $\Xi$ the complex crown [9].

Let us mention that the image of $T_{t}$ can be very different in nature: a weighted Bergman space for $M=\mathbb{R}^{n}, U, U / K$, a sum of two weighted Bergman spaces with oscillatory weight for $M=\mathbb{H}$, and, finally, for $M=$ $G / K$ the image is not a Bergman space at all and needs to be characterized with tools from integral geometry.

We observe, that in all so far understood examples $M$ is a symmetric space. Hence one might ask if it is also possible to characterize $\operatorname{im} T_{t}$ for $M$ a locally symmetric space. This paper constitutes a modest step in that direction when we consider nilmanifolds associated to the Heisenberg group.

To be precise, for $M=\Gamma \backslash \mathbb{H}$ with $\Gamma<\mathbb{H}$ the standard lattice we show that $\operatorname{im} T_{t}$ is the sum of three weighted Bergman spaces two of which with oscillatory weight. To establish this theorem we lean on results for $\mathbb{H}$ [8] as well as on deeper facts on the Hermite semigroup [4]. Finally, let us mention that most of our methods extend to arbitrary discrete subgroups $\Gamma<\mathbb{H}$.

Acknowledgments. The authors wish to thank the referee for making several useful comments on an earlier version of the paper.

\section{Nilmanifolds associated to the Heisenberg group and the heat kernel transform}

\subsection{Nilmanifolds associated to the Heisenberg group}

Let us denote by $\mathbb{H}$ the $(2 n+1)$-dimensional Heisenberg group. As a manifold $\mathbb{H}=\mathbb{R}^{n} \times \mathbb{R}^{n} \times \mathbb{R}$ and the group law is given by

$$
(x, u, t)\left(x^{\prime}, u^{\prime}, t^{\prime}\right)=\left(x+x^{\prime}, u+u^{\prime}, t+t^{\prime}+\frac{1}{2}\left(u \cdot x^{\prime}-x \cdot u^{\prime}\right)\right) .
$$

Throughout $\Gamma$ will denote a discrete subgroup of $\mathbb{H}$. With this data we form the nilmanifold $\Gamma \backslash \mathbb{H}$. Sometimes we abbreviate $M=\Gamma \backslash \mathbb{H}$. Here are some examples of $\Gamma$ we have in mind:

Examples: (a) Let $\Gamma=\{0\} \times\{0\} \times \mathbb{Z}$. Then $\Gamma<\mathbb{H}$ is a discrete central subgroup. The quotient $\mathbb{H}_{\text {red }}=\Gamma \backslash \mathbb{H}$ is the familiar reduced Heisenberg group.

(b) The choice $\Gamma_{\text {st }}=\mathbb{Z}^{n} \times \mathbb{Z}^{n} \times \frac{1}{2} \mathbb{Z}$ defines the standard lattice in $\mathbb{H}$. (This terminology is not standard!). The quotient $\Gamma_{\text {st }} \backslash \mathbb{H}$ is a non-trivial 
circle bundle over the $2 n$-torus $\mathbb{T}^{2 n}$ and hence compact. The fundamental group of this compact manifold is the non-Abelian group $\Gamma_{\text {st. }}$.

(c) The prescription $\Gamma=\mathbb{Z}^{n} \times\{0\} \times\{0\}$ defines an abelian discrete subgroup which does not intersect the center of $\mathbb{H}$.

For more about lattices in $\mathbb{H}$, especially their classification, we refer to [3], [5] and [13]. Write $N$ for the smallest connected subgroup containing $\Gamma$ ( $N$ coincides with the Zariski-closure of $\Gamma$ in the algebraic group $\mathbb{H}$ (cf. [10, Ch. II, Remark 2.6]). We notice that $\Gamma$ becomes a lattice in the nilpotent group $N$.

Write $\mathfrak{n}$ for the Lie algebra of $N$. Then we find a subspace $\mathfrak{v} \subset \mathfrak{h}$ such that $\mathfrak{h}=\mathfrak{n}+\mathfrak{v}$. The prescription $V=\exp (\mathfrak{v})$ defines a closed submanifold of $\mathbb{H}$ and the multiplication mapping

$$
N \times V \rightarrow \mathbb{H}, \quad(n, v) \mapsto n v
$$

is a polynomial homeomorphism. Consequently we obtain

$$
\Gamma \backslash \mathbb{H} \simeq \Gamma \backslash N \times \mathfrak{v},
$$

where $\Gamma \backslash N$ is compact and $\mathfrak{v}$ is a vector space.

Write $d h$ for a Haar-measure on $\mathbb{H}$ which we normalize such that it coincides with the Lebesgue measure once we identify $\mathbb{H}$ with $\mathbb{R}^{2 n+1}$. Denote by $d(\Gamma h)$ the unique measure on $\Gamma \backslash \mathbb{H}$ which satisfies

$$
\int_{\mathbb{H}} f(h) d h=\int_{\Gamma \backslash \mathbb{H}} \sum_{\gamma \in \Gamma} f(\gamma h) d(\Gamma h)
$$

for all $f \in C_{c}(\mathbb{H})$. The corresponding $L^{p}$-spaces shall be denoted by $L^{p}(\Gamma \backslash \mathbb{H})$.

An important tool for us will be the averaging operator

$$
A: C_{c}(\mathbb{H}) \rightarrow C_{c}(\Gamma \backslash \mathbb{H}), f \mapsto A(f) ; A(f)(\Gamma h)=\sum_{\gamma \in \Gamma} f(\gamma h)
$$

which is known to be continuous and onto. Observe that $A$ naturally extends to a surjective contraction $L^{1}(\mathbb{H}) \rightarrow L^{1}(\Gamma \backslash \mathbb{H})$, also denoted by $A$. Likewise (2.1) implies that $A$ induces a continuous surjection of Schwartz spaces $S(\mathbb{H}) \rightarrow S(\Gamma \backslash \mathbb{H})$.

\subsection{Definition and basic properties of the heat kernel transform on $\Gamma \backslash \mathbb{H}$}

The universal complexification of $\mathbb{H}_{\mathbb{C}}$ of $\mathbb{H}$ is simply $\mathbb{H}_{\mathbb{C}}=\mathbb{C}^{n} \times \mathbb{C}^{n} \times \mathbb{C}$ with holomorphically extended group law

$$
(z, w, \zeta)\left(z^{\prime}, w^{\prime}, \zeta^{\prime}\right)=\left(z+z^{\prime}, w+w^{\prime}, \zeta+\zeta^{\prime \prime}+\frac{1}{2}\left(w \cdot z^{\prime}-z \cdot w^{\prime}\right)\right) .
$$

Here $z \cdot w=\sum_{j=1}^{n} z_{j} w_{j}$. 
If a real analytic function $f$ on $\mathbb{H}$ admits holomorphic extension to $\mathbb{H}_{\mathbb{C}}$, then $\tilde{f}$ shall be the notation for this so obtained function.

We write $\Delta$ for the standard left Laplacian on $\mathbb{H}$ (cf. [8, Sect. 2.2]) and denote by $k_{t}$ the corresponding heat kernel on $\mathbb{H}$. Explicitly

$$
k_{t}(x, u, \xi)=c_{n} \int_{-\infty}^{\infty} e^{-i \lambda \xi} e^{-t \lambda^{2}}\left(\frac{\lambda}{\sinh (\lambda t)}\right)^{n} e^{-\frac{1}{4} \lambda \operatorname{coth}(\lambda t)\left(x^{2}+u^{2}\right)} d \lambda
$$

where $c_{n}$ is a constant and $x^{2}=\sum_{j=1}^{n} x_{j}^{2}, u^{2}=\sum_{j=1}^{n} u_{j}^{2}$ (cf. [8, Sect. 2.2]). From the above it is clear that $k_{t}$ can be extended to a holomorphic function on $\mathbb{H}_{\mathbb{C}}$, namely

$$
k_{t}^{\sim}(z, w, \zeta)=c_{n} \int_{-\infty}^{\infty} e^{-i \lambda \zeta} e^{-t \lambda^{2}}\left(\frac{\lambda}{\sinh (\lambda t)}\right)^{n} e^{-\frac{1}{4} \lambda \operatorname{coth}(\lambda t)\left(z^{2}+w^{2}\right)} d \lambda .
$$

As before $z^{2}=\sum_{j=1}^{n} z_{j}^{2}$ and $w^{2}=\sum_{j=1}^{n} w_{j}^{2}$. Note that $k_{t}^{\sim}$ is well behaved in the sense that its restriction to any bi-translate $g \mathbb{H} h \subset \mathbb{H}_{\mathbb{C}}$ with $h, g \in \mathbb{H}_{\mathbb{C}}$ is of rapid decay, i.e. $\mathbb{H} \ni x \mapsto k_{t}^{\sim}(g x h)$ is in $\mathcal{S}(\mathbb{H})$.

For $t>0$ the heat kernel transform $T_{t}$ on $\mathbb{H}$ is defined by

$$
T_{t}: L^{2}(\mathbb{H}) \rightarrow \mathcal{O}\left(\mathbb{H}_{\mathbb{C}}\right), \quad f \mapsto\left(f * k_{t}\right)^{\sim}
$$

and one immediately verifies that

- $T_{t}$ is continuous (with $\mathcal{O}\left(\mathbb{H}_{\mathbb{C}}\right)$ carrying the Fréchet topology of compact convergence),

- $T_{t}$ is injective,

- $T_{t}$ is left- $\mathbb{H}$ equivariant

(see [8, Sect. 3.1]).

It is not hard to see that $T_{t}$ extends to a map on tempered distributions

$$
\overline{T_{t}}: \mathcal{S}^{\prime}(\mathbb{H}) \rightarrow \mathcal{O}\left(\mathbb{H}_{\mathbb{C}}\right), \quad \nu \mapsto\left(\nu * k_{t}\right)^{\sim}
$$

featuring the bulleted items from above. With that we turn to the heat kernel transform on the nil-manifold $M=\Gamma \backslash \mathbb{H}$. We often identify functions on $\Gamma \backslash \mathbb{H}$ with $\Gamma$-invariant functions on $\mathbb{H}$. In this way we have $L^{2}(\Gamma \backslash \mathbb{H}) \subset$ $\mathcal{S}^{\prime}(\mathbb{H})$ and with $T_{t}^{\Gamma}=\left.\bar{T}_{t}\right|_{L^{2}(\Gamma \backslash \mathbb{H})}$ we obtain a continuous injection

$$
T_{t}^{\Gamma}: L^{2}(\Gamma \backslash M) \rightarrow \mathcal{O}\left(\Gamma \backslash \mathbb{H}_{\mathbb{C}}\right) .
$$

Remark 2.1 Recall that $\Delta$ was defined by the use of left-invariant vector fields on $\mathbb{H}$ and so factors to the Laplacian $\Delta^{\Gamma}$ on the nilmanifold $\Gamma \backslash \mathbb{H}$. It is easy to see that

$$
\left(e^{t \Delta^{\Gamma}} f\right)^{\sim}=T_{t}^{\Gamma}(f)
$$

for all $f \in L^{2}(\Gamma \backslash \mathbb{H})$. 
It is useful to have an alternative way to describe $T_{t}^{\Gamma}$. Invoking the decomposition (2.1) and the spectral resolution of $k_{t}^{\sim}(\mathrm{cf} .(2.2))$ it is not hard to see that series

$$
K_{t}^{\Gamma}(\Gamma h, \Gamma z)=\sum_{\gamma \in \Gamma} k_{t}^{\sim}\left(h^{-1} \gamma w\right) \quad\left(h \in \mathbb{H}, z \in \mathbb{H}_{\mathbb{C}}\right)
$$

converges uniformly in the first and locally uniformly in the second variable. As a result

$$
K_{t}^{\Gamma} \in \mathcal{S}\left(\Gamma \backslash \mathbb{H}_{\mathbb{C}}\right) \hat{\otimes} \mathcal{O}\left(\Gamma \backslash \mathbb{H}_{\mathbb{C}}\right)
$$

We observe

$$
\left(T_{t}^{\Gamma} f\right)(\Gamma z)=\int_{\Gamma \backslash \mathbb{H}} f(\Gamma h) K_{t}^{\Gamma}(\Gamma h, \Gamma z) d(\Gamma h)
$$

and deduce the inequality:

Lemma 2.2 Let $Q \subseteq \mathbb{H}_{\mathbb{C}}$ be a compact subset. Then

$$
C:=C(Q):=\sup _{z \in Q}\left\|K_{t}^{\Gamma}(\cdot, \Gamma z)\right\|_{L^{2}(\Gamma \backslash \mathbb{H})}<\infty
$$

and one has

$$
\sup _{z \in Q}\left|T_{t}^{\Gamma}(f)(\Gamma z)\right| \leq C\|f\|_{L^{2}(\Gamma \backslash \mathbb{H})} .
$$

We conclude this subsection with the averaging-equivariance.

Lemma 2.3 Let $f \in \mathcal{S}(\Gamma \backslash \mathbb{H})$ and $F \in \mathcal{S}(\mathbb{H})$ such that $A(F)=f$. Then

$$
T_{t}^{\Gamma}(f)=A\left(H_{t}(F)\right) .
$$

Proof. For all $z \in \mathbb{H}_{\mathbb{C}}$ we have

$$
\begin{aligned}
T_{t}^{\Gamma}(f)(\Gamma z) & =\int_{\mathbb{H}} f(h) k_{t}\left(h^{-1} z\right) d h \\
& =\sum_{\gamma \in \Gamma} \int_{\mathbb{H}} F(\gamma h) k_{t}\left(h^{-1} z\right) d h \\
& =\sum_{\gamma \in \Gamma} \int_{\mathbb{H}} F(h) k_{t}\left(h^{-1} \gamma z\right) d h \\
& =A\left(T_{t}(F)\right)(\Gamma z),
\end{aligned}
$$

as was to be shown. 


\section{3. $\Gamma$-invariant distribution vectors and the Plancherel- Theorem for $L^{2}(M)$}

Throughout this section and the next we confine ourselves to the standard lattice

$$
\Gamma=\Gamma_{\mathrm{st}}=\mathbb{Z}^{n} \times \mathbb{Z}^{n} \times \frac{1}{2} \mathbb{Z} .
$$

We will classify the $\Gamma$-invariant distribution vectors for the Schrödinger representation and relate this to the Plancherel decomposition of $L^{2}(M)$.

We wish to point out that the material collected below is all well known, see [6], [1], [3], [13] and especially [5] for a particularly nice treatment. The decomposition of $L^{2}(M)$ into irreducible pieces is due to Brezin [3] and we refer to [5] for an explicit exposition. In the terminology of [5] the lattice $\Gamma_{\mathrm{st}}$ is isomorphic to $\Gamma_{l}$ where $l=(2,2, \ldots, 2)$ via the automorphism $(x, u, t) \rightarrow(x, 2 u, 2 t)$.

In our exposition we adapt the more general point of view of Gelfand et al. which, in our opinion, clarifies the underlying structure best.

To begin with we consider a unimodular Lie group $G$ and let $\Gamma<G$ be a co-compact lattice. Form $M=\Gamma \backslash G$. One is interested in decomposing the right-regular representation $R$ on $L^{2}(M)$ into irreducibles. In this context one has a basic result (cf. [6, Ch. 1 , Sect. 4.6])

$$
L^{2}(M) \simeq \bigoplus_{\pi \in \hat{G}} m(\pi) \mathcal{H}_{\pi} .
$$

Here, as usual, $\hat{G}$ denotes the unitary dual of $G$ and the multiplicities $m(\pi)$ are all finite. By Frobenius reciprocity one has

$$
m(\pi)=\operatorname{dim}_{\mathbb{C}}\left(\mathcal{H}_{\pi}^{-\infty}\right)^{\Gamma},
$$

where $\left(\mathcal{H}_{\pi}^{-\infty}\right)^{\Gamma}$ denotes the space of $\Gamma$-invariant distribution vectors of $\left(\pi, \mathcal{H}_{\pi}\right)$. Let us make the unitary equivalence in (3.1) explicit by writing down the intertwining operator. There exists an inner product $\langle,\rangle_{\pi}$ on the finite dimensional $\mathbb{C}$-vectorspace $\mathbf{M}_{\pi}=\left(\mathcal{H}_{\pi}^{-\infty}\right)^{\Gamma}$ such that the map

$$
\sum_{\pi \in \hat{G}} \mathbf{M}_{\pi} \otimes \mathcal{H}_{\pi}^{\infty} \rightarrow L^{2}(M), \quad \sum \nu_{\pi} \otimes v_{\pi} \mapsto\left(\Gamma g \mapsto \sum_{\pi} \nu_{\pi}\left(\pi(g) v_{\pi}\right)\right)
$$

extends to a unitary $G$-equivalence

$$
\bigoplus_{\pi \in \hat{G}} \mathbf{M}_{\pi} \otimes \mathcal{H}_{\pi} \simeq L^{2}(M) .
$$

Thus for our special situation $M=\Gamma \backslash \mathbb{H}$ we have to determine two things: first $\mathbf{M}_{\pi}$, and secondly the inner product $\langle\cdot, \cdot\rangle_{\pi}$ on this space. We turn to the details. 
The first step in the decomposition of $R$ is the Fourier decomposition of $f \in L^{2}(M)$ in the last variable. Note that $F(x, u, \xi)$ is $\frac{1}{2}$ - periodic in $\xi$ and hence it has the expansion

$$
F(x, u, \xi)=\sum_{k=-\infty}^{\infty} F^{k}(x, u) e^{4 \pi i k \xi}
$$

where $F^{k}(x, u)$ are the Fourier coefficients of $F(x, u, \xi)$. Thus $L^{2}(M)$ has the orthogonal direct sum decomposition

$$
L^{2}(M)=\sum_{k \in \mathbb{Z}} \mathcal{H}_{k}
$$

where $\mathcal{H}_{k}$ is the set of all functions $F \in L^{2}(M)$ satisfying $F(x, u, \xi)=$ $e^{4 \pi i k \xi} F(x, u, 0)$. We now proceed to obtain further decomposition of $\mathcal{H}_{k}$ for each $k \neq 0$.

Let $\pi_{\lambda}, \lambda \in \mathbb{R}, \lambda \neq 0$ be the Schrödinger representations of $\mathbb{H}$ realised on $L^{2}\left(\mathbb{R}^{n}\right)$. Explicitly,

$$
\pi_{\lambda}(x, u, \xi) \varphi(v)=e^{i \lambda \xi} e^{i \lambda\left(x \cdot v+\frac{1}{2} x \cdot u\right)} \varphi(v+u) .
$$

The subspaces $\mathcal{H}_{k}$ are invariant under $R$ and by Stone-von Neumann theorem $R$ restricted to $\mathcal{H}_{k}$ decomposes into finitely many pieces each equivalent to $\pi_{4 \pi k}$. For explicit decompositions of $R$ we refer to [3], [13] and [5]. There is no canonical way of effecting the decomposition and here we get one such decomposition which is suitable for our purpose of studying the heat kernel transform. For some 'natural' decompositions of $\mathcal{H}_{k}$ we refer to Auslander and Brezin [1].

As we already described earlier, the standard way of constructing $\Gamma$ invariant functions on $\mathbb{H}$ is to start with a tempered distribution $\nu$ on $\mathbb{R}^{n}$ which is $\pi_{\lambda}(\Gamma)$ invariant and consider $F(x, u, \xi)=\left(\nu, \pi_{\lambda}(x, u, \xi) f\right)$ where $f$ is a Schwartz function on $\mathbb{R}^{n}$. Let $\nu$ be such a distribution; that is it verifies $\left(\nu, \pi_{\lambda}(h) f\right)=(\nu, f), h \in \mathbb{H}$. Then taking $h=(0,0, j / 2) \in \Gamma, j \in \mathbb{Z}$ we are led to $\pi_{\lambda}(h) f=e^{i \lambda j / 2} f$ and $\left(\nu, e^{i \lambda j / 2} f\right)=(\nu, f)$. This holds for all $j \in \mathbb{Z}$ if and only if $\lambda=4 \pi k$ for some $k \in \mathbb{Z}$. Let us assume $k \neq 0$ and write $\rho_{k}=\pi_{4 \pi k}$.

Proposition 3.1 Set $A_{k}=\left\{\mathbf{j} \in \mathbb{Z}^{n}: 0 \leq j_{1}, j_{2}, \ldots, j_{n} \leq 2 k-1\right\}$. Then every tempered distribution $\nu$ invariant under $\rho_{k}(\Gamma)$ is of the form $\nu=$ $\sum_{\mathbf{j} \in A_{k}} c_{\mathbf{j}} \nu_{\mathbf{j}}$ with $\nu_{\mathbf{j}}$ defined by

$$
\left(\nu_{\mathbf{j}}, f\right)=\sum_{\mathbf{m} \in \mathbb{Z}^{n}} \hat{f}(2 k \mathbf{m}+\mathbf{j}) \quad(f \in \mathcal{S}(\mathbb{H}))
$$

Here $\hat{f}$ denotes the Fourier transform of the Schwartz class function $f$. 
Proof. Since $\lambda=4 k \pi, e^{i \lambda \xi}=1$ for $\xi \in \frac{1}{2} \mathbb{Z}$. The $\rho_{k}(\Gamma)$-invariance of $\nu$ shows that

$$
\left(\nu, \rho_{k}(0, \mathbf{n}, 0) f\right)=(\nu, f(\cdot+\mathbf{n}))=(\nu, f)
$$

which means that $\nu$ is periodic. Consequently, $\nu$ has the Fourier expansion

$$
(\nu, f)=\sum_{\mathbf{n} \in \mathbb{Z}^{n}} c_{\mathbf{n}} \hat{f}(\mathbf{n})
$$

where $\hat{f}(\eta)=\int_{\mathbb{R}^{n}} f(x) e^{-2 \pi i x \cdot \eta} d x$. The $\rho_{k}(\Gamma)$ invariance of $\nu$ also shows that

$$
(\nu, f)=\left(\nu, \rho_{k}(\mathbf{m}, 0,0) f\right)=\left(\nu, e^{4 \pi k i \mathbf{m} \cdot(\cdot)} f\right)
$$

which translates into

$$
\sum_{\mathbf{n} \in \mathbb{Z}^{n}} c_{\mathbf{n}} \hat{f}(\mathbf{n}-2 k \mathbf{m})=\sum_{\mathbf{n} \in \mathbb{Z}^{n}} c_{\mathbf{n}} \hat{f}(\mathbf{n})
$$

This shows that $c_{\mathbf{n}}$ is a constant on the equivalence classes in $\mathbb{Z}^{n} / 2 k \mathbb{Z}^{n}$. Thus

$$
(\nu, f)=\sum_{\mathbf{j} \in A_{k}} c_{\mathbf{j}} \sum_{\mathbf{m} \in \mathbb{Z}^{n}} \hat{f}(2 k \mathbf{m}+\mathbf{j})
$$

Defining $\nu_{\mathbf{j}}$ accordingly we complete the proof.

Remark 3.2 In view of the Poisson summation formula

$$
\sum_{\mathbf{m} \in \mathbb{Z}^{n}} f(x+\mathbf{m})=\sum_{\mathbf{m} \in \mathbb{Z}^{n}} \hat{f}(\mathbf{m}) e^{2 \pi i \mathbf{m} \cdot x},
$$

valid for all functions $f \in \mathcal{S}\left(\mathbb{R}^{n}\right)$, we have

$$
\left(\nu_{\mathbf{j}}, f\right)=(2 k)^{-n} \sum_{\mathbf{m} \in \mathbb{Z}^{n}} e^{-\frac{\pi i}{k} \mathbf{m} \cdot \mathbf{j}} f\left(\frac{1}{2 k} \mathbf{m}\right) .
$$

Remark 3.3 Note that $\mathcal{S}\left(\mathbb{R}^{n}\right)$ is the space of smooth vectors for the Schrödinger representation $\rho_{k}$, i.e.

$$
L^{2}\left(\mathbb{R}^{n}\right)^{\infty}=\mathcal{S}\left(\mathbb{R}^{n}\right)
$$

in the standard representation theory terminology. Dualizing this identity we obtain

$$
L^{2}\left(\mathbb{R}^{n}\right)^{-\infty}=\mathcal{S}^{\prime}\left(\mathbb{R}^{n}\right)
$$

and with it the Gelfand-triplet

$$
L^{2}\left(\mathbb{R}^{n}\right)^{\infty}=\mathcal{S}\left(\mathbb{R}^{n}\right) \hookrightarrow L^{2}\left(\mathbb{R}^{n}\right) \hookrightarrow \mathcal{S}^{\prime}\left(\mathbb{R}^{n}\right)=L^{2}\left(\mathbb{R}^{n}\right)^{-\infty} .
$$

The above proposition then implies that

$$
\operatorname{dim}_{\mathbb{C}}\left(L^{2}\left(\mathbb{R}^{n}\right)^{-\infty}\right)^{\Gamma}=(2 k)^{n}
$$

and, moreover, provides an explicit basis for the space $\left(L^{2}\left(\mathbb{R}^{n}\right)^{-\infty}\right)^{\Gamma}$. 
At this point it might be interesting to observe that there is an alternative way to construct elements of $\left(L^{2}\left(\mathbb{R}^{n}\right)^{-\infty}\right)^{\Gamma}$, namely by averaging: Let $f \in$ $\mathcal{S}\left(\mathbb{R}^{n}\right)$. It is not difficult to show that the series

$$
\sum_{\gamma \in \Gamma} \rho_{k}(\gamma)(f)
$$

converges in $\mathcal{S}^{\prime}\left(\mathbb{R}^{n}\right)$ and defines a $\Gamma$-invariant element there. One establishes that the map

$$
S(\mathbb{R})^{n} \rightarrow \mathcal{S}^{\prime}\left(\mathbb{R}^{n}\right)^{\Gamma}, \quad f \mapsto \sum_{\gamma \in \Gamma} \rho_{k}(\gamma)(f)
$$

is a continuous surjection.

At this point we determined the spectrum of $L^{2}(M)$, i.e. the occuring unitary irreducible representations, as well as the multiplicity space $\mathbf{M}_{\pi}$. In the sequel we abbreviate $\mathbf{M}_{k}:=\mathbf{M}_{\rho_{k}}$. As a last step we have to determine the unitary structure on $\mathbf{M}_{k}$ such that the map $f \rightarrow\left(\nu_{\mathbf{j}}, \rho_{k}(\cdot) f\right)$ becomes isometric. We already know that

$$
\mathbf{M}_{k}=\operatorname{span}\left\{\nu_{\mathbf{j}} \mid \mathbf{j} \in A_{k}\right\}
$$

and in the next step we want to show that the $\nu_{\mathbf{j}}$ are in fact orthogonal. This is easy and follows from a little group theory. In fact, let us define the finite group

$$
\mathbf{F}_{k}:=(\mathbb{Z} / 2 k \mathbb{Z})^{n}
$$

Then the prescription

$$
\Pi_{k}(x)(\nu):=\nu(\cdot+x) \quad\left(\nu \in \mathbf{M}_{k}, x \in \mathbf{F}_{k}\right)
$$

defines a representation of $\mathbf{F}_{k}$ on $\mathbf{M}_{k}$. Moreover it is clear that $\nu_{\mathbf{j}}$ is a basis of eigenvectors for this action; explicitly:

$$
\Pi_{k}(x) \nu_{\mathbf{j}}=e^{\frac{\pi i}{k} x \cdot \mathbf{j}} \nu_{\mathbf{j}} \quad\left(\mathbf{j} \in A_{k}\right) .
$$

Furthermore for $f, g \in L^{2}\left(\mathbb{R}^{n}\right)$ and $\nu, \mu \in \mathbf{M}_{k}$ we set

$$
F_{\nu}(x, u, \xi)=\left(\nu, \rho_{k}(x, u . \xi) f\right) \quad \text { and } \quad G_{\mu}(x, u, \xi)=\left(\mu, \rho_{k}(x, u, \xi) g\right) .
$$

Then one immediately verifies that

$$
\left(F_{\Pi_{k}(x) \nu}, G_{\mu}\right)_{L^{2}(M)}=\left(F_{\nu}, G_{\Pi_{k}(-x) \mu}\right)_{L^{2}(M)} .
$$

Thus we conclude with (3.4) that $\nu_{\mathbf{j}}$ is in fact an orthogonal basis (up to an uniform scalar) for $\mathbf{M}_{k}$. Furthermore it is indeed an orthonormal basis, as the next Lemma will show. 
Lemma 3.4 Let $f \in L^{2}\left(\mathbb{R}^{n}\right), \mathbf{j} \in A_{k}$ and $F \in L^{2}(M)$ defined by the corresponding matrix coefficient

$$
F(x, u, \xi)=\left(\nu_{\mathbf{j}}, \rho_{k}(x, u, \xi) f\right)
$$

Then

$$
\|F\|_{L^{2}(M)}=\sqrt{2} \cdot\|f\|_{L^{2}\left(\mathbb{R}^{n}\right)} .
$$

Proof. The proof is a straightforward computation; we simply expand the terms:

$$
\begin{aligned}
F(x, u, \xi) & =\left(\nu_{\mathbf{j}}, \rho_{k}(x, u, \xi) f\right) \\
& =\left(\nu_{\mathbf{j}}, e^{4 \pi i k \xi} e^{4 \pi i k\left(x \cdot+\frac{1}{2} x \cdot u\right)} f(\cdot+u)\right) \\
& =e^{4 \pi i k \xi} e^{2 \pi i x \cdot u}\left(\nu_{\mathbf{j}}, e^{4 \pi i k x \cdot \cdot} f(\cdot+u)\right) \\
& =\frac{1}{(2 k)^{n}} e^{4 \pi i k \xi} e^{2 \pi i x \cdot u} \sum_{\mathbf{m} \in \mathbb{Z}^{n}} e^{-\frac{\pi i}{k} \mathbf{m} \cdot \mathbf{j}} e^{2 \pi i x \cdot \mathbf{m}} f\left(\frac{1}{2 k} \mathbf{m}+u\right) .
\end{aligned}
$$

In the last equation we used the characterization of $\nu_{j}$ from Remark 3.2. As $\Gamma \backslash \mathbb{H}$ coincides with $\mathbb{R} / \frac{1}{2} \mathbb{Z} \times \mathbb{R}^{n} / \mathbb{Z}^{n} \times \mathbb{R}^{n} / \mathbb{Z}^{n}$ up to set of measure zero, we thus get

$$
\begin{aligned}
\|F\|_{L^{2}(M)}^{2} & =\frac{2}{(2 k)^{2 n}} \int_{\mathbb{R}^{n} / \mathbb{Z}^{n}} \int_{\mathbb{R}^{n} / \mathbb{Z}^{n}}\left|\sum_{\mathbf{m} \in \mathbb{Z}^{n}} e^{-\frac{\pi i}{k} \mathbf{m} \cdot \mathbf{j}} e^{2 \pi i x \cdot \mathbf{m}} f\left(\frac{1}{2 k} \mathbf{m}+u\right)\right|^{2} \\
& =\frac{2}{(2 k)^{2 n}} \int_{\mathbb{R}^{n} / \mathbb{Z}^{n}} \sum_{\mathbf{m} \in \mathbb{Z}^{n}}\left|f\left(\frac{1}{2 k} \mathbf{m}+u\right)\right|^{2} d u \\
& =\frac{2}{(2 k)^{n}} \int_{\mathbb{R}^{n} / \mathbb{Z}^{n}} \sum_{\mathbf{m} \in \mathbb{Z}^{n}}\left|f\left(\frac{1}{2 k}(\mathbf{m}+u)\right)\right|^{2} d u \\
& =\frac{2}{(2 k)^{n}} \int_{\mathbb{R}^{n}}\left|f\left(\frac{1}{2 k} u\right)\right|^{2} d u \\
& =2\|f\|^{2},
\end{aligned}
$$

as was to be shown.

Finally we make some remarks to the existing literature. We show that the matrix coefficients $F_{\mathbf{j}}(x, u, \xi):=\left(\nu_{\mathbf{j}}, \rho_{k}(x, u, \xi) f\right)$ can be expressed as Weil- Brezin (or Zak ) transforms studied in [3]. Consider the operator $V_{k}$ defined on the Schwartz class $\mathcal{S}\left(\mathbb{R}^{n}\right)$ by

$$
V_{k} f(x, u, \xi)=\sum_{\mathbf{m} \in \mathbb{Z}^{n}} \rho_{k}(x, u, \xi) f(\mathbf{m}) .
$$


Written explicitly

$$
V_{k} f(x, u, \xi)=e^{4 \pi k i \xi} e^{2 \pi k i x \cdot u} \sum_{\mathbf{m} \in \mathbb{Z}^{n}} e^{4 \pi k i \mathbf{m} \cdot x} f(u+\mathbf{m}) .
$$

It is easy to see that $V_{k} f$ is $\Gamma$ invariant. For each $\mathbf{j} \in A_{k}$ we also define

$$
V_{k, \mathbf{j}} f(x, u, \xi)=e^{2 \pi i \mathbf{j} \cdot x} V_{k} f(x, u, \xi) .
$$

These are called the Weil-Brezin transforms in the literature.

Proposition 3.5 1. For each $f \in \mathcal{S}\left(\mathbb{R}^{n}\right)$ we have the relation

$$
F_{\mathbf{j}}(x, u, \xi)=V_{k, \mathbf{j}} g_{\mathbf{j}}(u,-x, \xi)
$$

where $f$ and $g_{\mathbf{j}}$ are related by $g_{\mathbf{j}}(x)=\hat{f}(2 k x+\mathbf{j})$.

2. The transform $V_{k, \mathbf{j}}$ initially defined on $\mathcal{S}\left(\mathbb{R}^{n}\right)$ extends to the whole of $L^{2}\left(\mathbb{R}^{n}\right)$ as an isometry into $\mathcal{H}_{k}$.

Proof. To prove the first assertion, a simple calculation shows that

$$
\left(\rho_{k}(x, u, \xi) f \hat{)}(s)=e^{4 \pi k i \xi} e^{-2 \pi k i x \cdot u} e^{2 \pi i s \cdot u} \hat{f}(s-2 k x) .\right.
$$

Hence it follows that $F_{\mathbf{j}}(x, u, \xi)$ is given by

$$
e^{4 \pi k i \xi} e^{-2 \pi k i x \cdot u} \sum_{\mathbf{m} \in \mathbb{Z}^{n}} e^{2 \pi i(2 k \mathbf{m}+\mathbf{j}) \cdot u} \hat{f}(2 k \mathbf{m}+\mathbf{j}-2 k x)
$$

which simplifies to

$$
e^{4 \pi k i \xi} e^{-2 \pi k i x \cdot u} e^{2 \pi i \mathbf{j} \cdot u} \sum_{\mathbf{m} \in \mathbb{Z}^{n}} e^{4 \pi i \mathbf{m} \cdot u} \hat{f}(2 k(\mathbf{m}-x)+\mathbf{j}) .
$$

Setting $g_{\mathbf{j}}(s)=\hat{f}(2 k s+\mathbf{j})$ and recalling the definition of $V_{k, \mathbf{j}}$ we get

$$
F_{\mathbf{j}}(x, u, \xi)=V_{k, \mathbf{j}} g_{\mathbf{j}}(u,-x, \xi) .
$$

This shows that $\left(\nu_{\mathbf{j}}, \rho_{k}(x, u, \xi) f\right)$ can also be defined on the whole of $L^{2}\left(\mathbb{R}^{n}\right)$. Finally, notice that the second assertion follows from the first one and our preceding discussion.

Combining the two preceding propositions we get the following decomposition of the spaces $\mathcal{H}_{k}$.

Proposition 3.6 Let $\mathcal{H}_{k, \mathbf{j}}$ be the span of functions of the form

$$
F_{\mathbf{j}}=\left(\nu_{\mathbf{j}}, \rho_{k}(\cdot) f\right) \quad \text { as } f \text { varies over } L^{2}\left(\mathbb{R}^{n}\right) \text {. }
$$

Then $\mathcal{H}_{k}$ is the orthogonal direct sum of the spaces $\mathcal{H}_{k, \mathbf{j}}, \mathbf{j} \in A_{k}$. 
Remark 3.7 From the above proposition it follows that the restriction of $R$ to $\mathcal{H}_{k, \mathbf{j}}$ is unitarily equivalent to $\rho_{k}$. The intertwining operator is given by

$$
U_{k, \mathbf{j}} f(x, u, \xi)=\left(\nu_{\mathbf{j}}, \rho_{k}(x, u, \xi) f\right)
$$

which is also equal to the composition of $V_{k, \mathbf{j}}$ with the operators $f(s) \rightarrow$ $\hat{f}(2 k s+\mathbf{j})$ and $F(x, u, \xi) \rightarrow F(u,-x, \xi)$.

An orthonormal basis for $\mathcal{H}_{k}$ can be obtained using the operators $U_{k, \mathbf{j}}$. Let $\Phi_{\alpha}, \alpha \in \mathbb{N}^{n}$ be the normalised Hermite functions on $\mathbb{R}^{n}$. Then the functions $U_{k, \mathbf{j}} \Phi_{\alpha}(x, u, \xi)$ form an orthogonal system in $\mathcal{H}_{k, \mathbf{j}}$. With suitable normalising constants $c_{\alpha, \mathbf{j}}$ the functions $c_{\alpha, \mathbf{j}} U_{k, \mathbf{j}} \Phi_{\alpha}$ as $\alpha$ ranges over $\mathbb{N}^{n}$ and $\mathbf{j} \in A_{k}$ form an orthonormal basis for $\mathcal{H}_{k}$.

\section{The image of the heat kernel transform}

In this section we determine the image of $T_{t}^{\Gamma}$ for $\Gamma=\Gamma_{\text {st }}$ the standard lattice. To simplify notation we often write $T_{t}$ instead of $T_{t}^{\Gamma}$ and drop the $\sim$ for the holomorphic extension of a function.

As $L^{2}(M)$ is the direct sum of $\mathcal{H}_{k}$ as $k$ ranges over all integers, the image of $L^{2}(M)$ under $T_{t}$ will be the direct sum of $T_{t}\left(\mathcal{H}_{k}\right)$, the image of $\mathcal{H}_{k}$ under $T_{t}$. We first settle the case $k=0$. Recall that functions $f \in \mathcal{H}_{0}$ are independent of $\xi$ and hence we think of them as functions on the $2 n$-torus $\mathbb{T}^{n} \times \mathbb{T}^{n}$. An easy calculation (use (2.2)) shows that the function $f * k_{t}$ is given by the ordinary convolution

$$
f * k_{t}(z, w)=c_{n} t^{-n} \int_{\mathbb{R}^{n}} \int_{\mathbb{R}^{n}} f\left(x^{\prime}, u^{\prime}\right) e^{-\frac{1}{4 t}\left(\left(z-x^{\prime}\right)^{2}+\left(w-u^{\prime}\right)^{2}\right)} d x^{\prime} d u^{\prime} .
$$

Note that $f * k_{t}$ is an entire function on $\mathbb{C}^{n} \times \mathbb{C}^{n}$ which satisfies $f * k_{t}(z+$ $\mathbf{m}, w+\mathbf{n})=f * k_{t}(z, w)$ for all $\mathbf{m}, \mathbf{n} \in \mathbb{Z}^{n}$. Thus the heat kernel transform when restricted to $\mathcal{H}_{0}$ is nothing but the heat kernel transform on the torus $\mathbb{T}^{n} \times \mathbb{T}^{n}$ and the image has been characterised.

Theorem 4.1 An entire function $F(z, w)$ of $2 n$ complex variables belongs to $T_{t}\left(\mathcal{H}_{0}\right)$ if and only if $F(z+\mathbf{m}, w+\mathbf{n})=F(z, w)$ for all $\mathbf{m}, \mathbf{n} \in \mathbb{Z}^{n}$ and

$$
\|F\|^{2}=\int_{\mathbb{R}^{2 n}} \int_{[0,1)^{2 n}}|F(z, w)|^{2} e^{-\frac{1}{2 t}\left(y^{2}+v^{2}\right)} d x d u d y d v<\infty .
$$

Moreover $\left\|T_{t}(f)\right\|=\|f\|$ for all $f \in \mathcal{H}_{0}$.

Thus the members of $T_{t}\left(\mathcal{H}_{0}\right)$ are precisely the functions from the classical weighted Bergman space associated to the standard Laplacian on $\mathbb{R}^{2 n}$ that are periodic in the real parts of the variables. 
We now consider the image of $\mathcal{H}_{k}$ for $k \neq 0$. For the description of $T_{t}\left(\mathcal{H}_{k}\right)$ we need to recall several facts about Hermite-Bergman spaces and twisted Bergman spaces. Given a nonzero $\lambda \in \mathbb{R}$ consider the kernel

$$
p_{t}^{\lambda}(x, u)=c_{n} \lambda^{n}(\sinh (\lambda t))^{-n} e^{-\frac{1}{4} \lambda \operatorname{coth}(\lambda t)\left(x^{2}+u^{2}\right)} .
$$

This kernel is related to $k_{t}$ via

$$
k_{t}^{\lambda}(x, u)=e^{-t \lambda^{2}} p_{t}^{\lambda}(x, u)
$$

where for a function $f(x, u, \xi)$ on the Heisenberg group we use the notation

$$
f^{\lambda}(x, u)=\int_{-\infty}^{\infty} f(x, u, \xi) e^{i \lambda \xi} d \xi .
$$

Given a function $f \in L^{2}\left(\mathbb{R}^{2 n}\right)$ the $\lambda$-twisted convolution

$$
f *_{\lambda} p_{t}^{\lambda}(x, u)=\int_{\mathbb{R}^{2 n}} f\left(x^{\prime}, u^{\prime}\right) p_{t}^{\lambda}\left(x-x^{\prime}, u-u^{\prime}\right) e^{-i \frac{\lambda}{2}\left(u \cdot x^{\prime}-x \cdot u^{\prime}\right)} d x^{\prime} d u^{\prime}
$$

extends to $\mathbb{C}^{2 n}$ as an entire function. This transform is called the twisted heat kernel transform and in [7] we have studied the image of $L^{2}\left(\mathbb{R}^{2 n}\right)$ under this transform.

The image turns out to be the twisted Bergman space $\mathcal{B}_{t}^{\lambda}$ which is defined as follows. An entire function $F(z, w)$ on $\mathbb{C}^{2 n}$ belongs to $\mathcal{B}_{t}^{\lambda}$ if and only if

$$
\int_{\mathbb{C}^{n}} \int_{\mathbb{C}^{n}}|F(z, w)|^{2} W_{t}^{\lambda}(z, w) d z d w<\infty
$$

where

$$
W_{t}^{\lambda}(x+i y, u+i v)=e^{\lambda(u \cdot y-v \cdot x)} p_{2 t}^{\lambda}(2 y, 2 v) .
$$

In [7] it has been shown that the image of $L^{2}\left(\mathbb{R}^{2 n}\right)$ under the twisted heat kernel transform is precisely $\mathcal{B}_{t}^{\lambda}$.

The connection between the twisted heat kernel transform and the heat kernel transform on the nilmanifold $M$ is the following. If $F \in \mathcal{H}_{k}$ then an easy calculation shows that

$$
F * k_{t}(x, u, \xi)=e^{-t(4 \pi k)^{2}} e^{4 \pi i k \xi} G *_{-4 \pi k} p_{t}^{-4 \pi k}(x, u)
$$

where $G(x, u)=F(x, u, 0)$. Thus we are led to consider $\lambda$-twisted convolution with $p_{t}^{\lambda}$. Observe that when $F \in \mathcal{H}_{k}$ the function $G(x, u)=F(x, u, 0)$ satisfies

$$
G(x+\mathbf{m}, u+\mathbf{n})=e^{2 \pi i k(u \cdot \mathbf{m}-x \cdot \mathbf{n})} G(x, u) .
$$

Thus the entire extension of $G *_{-4 \pi k} p_{t}^{-4 \pi k}$ also inherits the same property and we expect the image of $\mathcal{H}_{k}$ under the heat kernel transform to consist of entire functions of the form

$$
F(z, w, \zeta)=e^{4 \pi i k \zeta} G(z, w),
$$

where $G$ has the above transformation property under translation by $\mathbb{Z}^{n} \times \mathbb{Z}^{n}$. 
We define $\mathcal{B}_{t, \Gamma}^{4 \pi k}$ to be the space of all entire functions $G(z, w)$ having the transformation property

$$
G(z+\mathbf{m}, w+\mathbf{n})=e^{2 \pi i k(w \cdot \mathbf{m}-z \cdot \mathbf{n})} G(z, w)
$$

which are square integrable with respect to $W_{t}^{-4 \pi k}$, i.e.

$$
\int_{\mathbb{R}^{2 n}} \int_{[0,1)^{2 n}}|G(z, w)|^{2} W_{t}^{-4 \pi k}(z, w) d x d u d y d v<\infty .
$$

\subsection{Diagonalization of $\mathcal{B}_{t, \Gamma}^{4 \pi k}$}

In this subsection we show that $\mathcal{B}_{t, \Gamma}^{4 \pi k}$ admits a natural symmetry of the finite group $\mathbf{F}_{k}$. To begin with we note that the prescription

$$
\left(\tilde{\Pi}_{k}(x) G\right)(z, w)=e^{-i \pi x \cdot w} G\left(z+\frac{x}{2 k}, w\right) \quad\left(x \in \mathbf{F}_{k}, G \in \mathcal{B}_{t, \Gamma}^{4 \pi k}\right)
$$

defines an action of $\mathbf{F}_{k}$ on $\mathcal{B}_{t, \Gamma}^{4 \pi k}$. Moreover,

Lemma 4.2 The representation $\left(\tilde{\Pi}_{k}, \mathcal{B}_{t, \Gamma}^{4 \pi k}\right)$ of $\mathbf{F}_{k}$ is unitary.

Proof. We have to show that

$$
\left\|\tilde{\Pi}_{k}(s) G\right\|^{2}=\|G\|^{2}
$$

for all $G \in \mathcal{B}_{t, \Gamma}^{4 \pi k}$ and $s \in \mathbf{F}_{k}$. The verification is a straightforward computation:

$$
\begin{aligned}
\left\|\tilde{\Pi}_{k}(s) G\right\|^{2}= & \int_{\mathbb{R}^{2 n}} \int_{[0,1)^{2 n}}\left|\left(\tilde{\Pi}_{k}(s) G\right)(x+i y, u+i v)\right|^{2} \\
& \times W_{t}^{-4 \pi k}(x+i y, u+i v) d x d u d y d v \\
= & \int_{\mathbb{R}^{2 n}} \int_{[0,1)^{2 n}}\left|\left(\tilde{\Pi}_{k}(s) G\right)(x+i y, u+i v)\right|^{2} \\
\times & \times e^{-4 \pi k(u \cdot y-v \cdot x)} p_{2 t}^{-4 \pi k}(2 y, 2 v) d x d u d y d v \\
= & \int_{\mathbb{R}^{2 n}} \int_{[0,1)^{2 n}}|G(x+s / 2 k+i y, u+i v)|^{2} \\
= & \int_{\mathbb{R}^{2 n}} \int_{[0,1)^{2 n}}|G(x+i y, u+i v)|^{2 \pi s \cdot v} e^{-4 \pi k(u \cdot y-v \cdot x)} p_{2 t}^{-4 \pi k}(2 y, 2 v) d x d u d y d v \\
= & \int_{\mathbb{R}^{2 n}} \int_{[0,1)^{2 n}}|G(x+i y, u+i v)|^{2 \pi s \cdot v} e^{-4 \pi k(u \cdot y-v \cdot(x-s / 2 k))} p_{2 t}^{-4 \pi k}(2 y, 2 v) d x d u d y d v \\
= & \quad \times e^{-4 \pi k(u \cdot y-v \cdot x)} p_{2 t}^{-4 \pi k}(2 y, 2 v) d x d u d y d v
\end{aligned}
$$


In the sequel we often abbreviate and write $\lambda$ for $4 \pi k$. Define for each $\mathbf{j} \in A_{k}$ a subspace $\mathcal{B}_{t, \mathbf{j}}^{\lambda}$ of $\mathcal{B}_{t, \Gamma}^{\lambda}$ as follows: $G \in \mathcal{B}_{t, \Gamma}^{\lambda}$ belongs to $\mathcal{B}_{t, \mathbf{j}}^{\lambda}$ if and only if

$$
G\left(z+\frac{1}{2 k} \mathbf{m}, w\right)=e^{\pi i \mathbf{m} \cdot\left(w+\frac{1}{k} \mathbf{j}\right)} G(z, w) .
$$

The previous lemma then implies:

Corollary 4.3 $\mathcal{B}_{t, \Gamma}^{\lambda}$ is the orthogonal direct sum of $\mathcal{B}_{t, \mathbf{j}}^{\lambda}, \mathbf{j} \in A_{k}$.

\subsection{Characterization of $\mathcal{B}_{t, \Gamma}^{\lambda}$}

The aim of this subsection is to prove that $T_{t}$ maps $\mathcal{H}_{k}$ onto $\mathcal{B}_{t, \Gamma}^{\lambda}$ (with $\mathcal{B}_{t, \Gamma}^{\lambda}$ interpreted as a subspace of $\mathcal{O}\left(\Gamma \backslash \mathbb{H}_{\mathbb{C}}\right)$ via (4.2)). Let us begin with the easy half, the isometry of the map. For that we have to introduce a useful technical tool, namely twisted averages.

Recall that $\mathcal{H}_{k}^{\infty}$ is the space of all functions $F$ in $\mathcal{S}(\Gamma \backslash \mathbb{H})$ which satisfy

$$
F(x, u, \xi)=e^{-i \lambda \xi} F(x, u, 0) .
$$

Often it is convenient to identify functions $f$ on $\mathbb{R}^{2 n}$ with functions $F$ on $\mathbb{H}$ which transform as (4.4) via

$$
f \leftrightarrow F, \quad F(x, u, \xi)=e^{-i \lambda \xi} f(x, u) .
$$

With this terminology we record for $F \in \mathcal{H}_{k}$

$$
T_{t}(F)(z, w, \zeta)=e^{i \lambda \zeta}\left(f *_{\lambda} p_{t}^{\lambda}\right)(z, w) .
$$

For $f \in \mathcal{S}\left(\mathbb{R}^{2 n}\right)$ we define its twisted average by

$$
A_{\lambda} f(x, u)=e^{i \lambda \xi} \sum_{\gamma \in \Gamma} F(\gamma(x, u, \xi))
$$

More explicitly,

$$
A_{\lambda} f(x, u)=\sum_{(\mathbf{a}, \mathbf{b}, 0) \in \Gamma} e^{i \frac{\lambda}{2}(u \cdot \mathbf{a}-x \cdot \mathbf{b})} f(x+\mathbf{a}, u+\mathbf{b}) .
$$

We note that $A_{\lambda}$ maps $\mathcal{S}\left(\mathbb{R}^{2 n}\right)$ onto $\mathcal{H}_{k}^{\infty}$ (modulo the identification (4.5)). Further we note that

$$
\left(A_{\lambda} f\right) *_{\lambda} p_{t}^{\lambda}=A_{\lambda}\left(f *_{\lambda} p_{t}^{\lambda}\right)
$$


Proposition 4.4 For all $f \in \mathcal{S}\left(\mathbb{R}^{2 n}\right)$ one has

$$
\left\|T_{t}\left(A_{\lambda}(f)\right)\right\|_{\mathcal{B}_{t, \Gamma}^{\lambda}}^{2}=\left\|A_{\lambda}(f)\right\|_{L^{2}(M)}^{2} .
$$

In particular, the map

$$
T_{t}: \mathcal{H}_{k} \rightarrow \mathcal{B}_{t, \Gamma}^{\lambda}, \quad F \mapsto f *_{\lambda} p_{t}^{\lambda}
$$

is isometric.

Proof. Let $f \in \mathcal{S}\left(\mathbb{R}^{2 n}\right)$. Then, by (4.8),

$$
\left(A_{\lambda} f\right) *_{\lambda} p_{t}^{\lambda}(z, w)=\sum_{(\mathbf{a}, \mathbf{b}, 0) \in \Gamma} e^{i \frac{\lambda}{2}(w \cdot \mathbf{a}-z \cdot \mathbf{b})} f *_{\lambda} p_{t}^{\lambda}(z+\mathbf{a}, w+\mathbf{b}) .
$$

We can obtain pointwise estimates for the function $f *_{\lambda} p_{t}^{\lambda}(z+\mathbf{a}, w+\mathbf{b})$ which shows that the above series actually converges. Therefore,

$$
\begin{aligned}
\left\|T_{t}\left(A_{\lambda}(f)\right)\right\|^{2} & =\int_{\mathbb{R}^{2 n}} \int_{\mathbb{R}^{2 n} / \mathbb{Z}^{2 n}}\left|\left(A_{\lambda} f\right) *_{\lambda} p_{t}^{\lambda}(z, w)\right|^{2} W_{t}^{\lambda}(z, w) d z d w \\
& =\int_{\mathbb{C}^{2 n}} f *_{\lambda} p_{t}^{\lambda}(z, w) \overline{\left(A_{\lambda} f\right) *_{\lambda} p_{t}^{\lambda}(z, w)} W_{t}^{\lambda}(z, w) d z d w
\end{aligned}
$$

and we used the transformation property of the weight function $W_{t}^{\lambda}$. Further expansion yields

$$
\begin{aligned}
\left\|T_{t}\left(A_{\lambda}(f)\right)\right\|^{2}= & \sum_{(\mathbf{a}, \mathbf{b}, 0) \in \Gamma} \int_{\mathbb{C}^{2 n}} f *_{\lambda} p_{t}^{\lambda}(z, w) . \\
& e^{-i \frac{\lambda}{2}(w \cdot \mathbf{a}-z \cdot \mathbf{b})} \overline{f *_{\lambda} p_{t}^{\lambda}}(z+\mathbf{a}, w+\mathbf{b}) W_{t}^{\lambda}(z, w) d z d w .
\end{aligned}
$$

We recall that $W_{t}^{\lambda}$ is the weight function for the twisted Bergman space $\mathcal{B}_{t}^{\lambda}$ (see [8]) and obtain further

$$
\begin{aligned}
\left\|T_{t}\left(A_{\lambda}(f)\right)\right\|^{2} & =\sum_{(\mathbf{a}, \mathbf{b}, 0) \in \Gamma} \int_{\mathbb{R}^{2 n}} f(x, u) e^{-i \frac{\lambda}{2}(u \cdot \mathbf{a}-x \cdot \mathbf{b})} \overline{f(x+\mathbf{a}, u+\mathbf{b})} d x d u \\
& =\int_{\mathbb{R}^{2 n} / \mathbb{Z}^{2 n}}\left|A_{\lambda} f(x, u)\right|^{2} d x d u \\
& =\left\|A_{\lambda} f\right\|^{2}
\end{aligned}
$$

which completes the proof.

We turn to the more difficult part, namely that $T_{t}$ maps $\mathcal{H}_{k}$ onto $\mathcal{B}_{t, \Gamma}^{\lambda}$. This will be proved by establishing a connection between twisted Bergman spaces and Hermite-Bergman spaces which we proceed to describe now. 
For each nonzero $\lambda \in \mathbb{R}$ let us consider the scaled Hermite operator $H(\lambda)=-\Delta+\lambda^{2}|x|^{2}$ on $\mathbb{R}^{n}$ whose eigenfunctions are provided by the Hermite functions

$$
\Phi_{\alpha}^{\lambda}(x)=|\lambda|^{\frac{n}{4}} \Phi_{\alpha}(\sqrt{|\lambda|} x), x \in \mathbb{R}^{n}, \alpha \in \mathbb{N}^{n} .
$$

The operator $H(\lambda)$ generates the Hermite semigroup $e^{-t H(\lambda)}$ whose kernel is explicitly given by

$$
K_{t}^{\lambda}(x, u)=\sum_{\alpha \in \mathbb{N}^{n}} e^{-(2|\alpha|+n)|\lambda| t} \Phi_{\alpha}^{\lambda}(x) \Phi_{\alpha}^{\lambda}(u)
$$

Using Mehler's formula (see [12]) the above series can be summed to get

$$
\begin{aligned}
K_{t}^{\lambda}(x, u)= & c_{n}(\sinh (\lambda t))^{-\frac{n}{2}}(\cosh (\lambda t))^{-\frac{n}{2}} \\
& \times e^{-\frac{\lambda}{4} \tanh (\lambda t)(x+u)^{2}} e^{-\frac{\lambda}{4} \operatorname{coth}(\lambda t)(x-u)^{2}} .
\end{aligned}
$$

The image of $L^{2}\left(\mathbb{R}^{n}\right)$ under the Hermite semigroup has been studied by Byun [4] . His result is stated as follows.

Let $\mathcal{H}_{t}^{\lambda}$ be the Hermite-Bergman space defined to be the space of all entire functions on $\mathbb{C}^{n}$ for which

$$
\int_{\mathbb{R}^{2 n}}|F(x+i y)|^{2} U_{t}^{\lambda}(x, y) d x d y<\infty
$$

where the weight function $U_{t}$ is given by

$$
U_{t}(x, y)=c_{n}(\sinh (4 \lambda t))^{-\frac{n}{2}} e^{\lambda \tanh (2 \lambda t) x^{2}} e^{-\lambda \operatorname{coth}(2 \lambda t) y^{2}} .
$$

Theorem 4.5 The image of $L^{2}\left(\mathbb{R}^{n}\right)$ under the Hermite semigroup is precisely the space $\mathcal{H}_{t}^{\lambda}$ and $e^{-t H(\lambda)}$ is a constant multiple of an isometry between these two spaces.

The relation between the heat kernel transform on $\Gamma \backslash \mathbb{H}$ and the Hermite semigroup is given in the following proposition.

Proposition 4.6 Let $f \in L^{2}\left(\mathbb{R}^{n}\right)$ and $F=V_{k, \mathbf{j}}(f)$ for $\mathbf{j} \in A_{k}$. Then

$$
F * k_{t}(x, u, \xi)=c_{\lambda} e^{-t \lambda^{2}+i \lambda \xi} e^{i \lambda\left(\mathbf{a} \cdot x+\frac{1}{2} x \cdot u\right)} \sum_{\mathbf{m} \in \mathbb{Z}^{n}} e^{i \lambda x \cdot \mathbf{m}} \tau_{-\mathbf{a}}\left(e^{-t H(\lambda)} \tau_{\mathbf{a}} f\right)(u+\mathbf{m})
$$

where $\lambda=4 \pi k, \mathbf{a}=\frac{1}{2 k} \mathbf{j}, \tau_{\mathbf{a}} f(x)=f(x-\mathbf{a})$ and $c_{\lambda}$ is a constant depending only on $\lambda$ and $n$. 
Proof. It follows from the definition of $V_{k, \mathbf{j}}$ and the calculation (3.1) that $e^{-i \lambda \xi+t \lambda^{2}} F * k_{t}(x, u, \xi)$ is given by

$$
\begin{aligned}
& \int_{\mathbb{R}^{2 n}} V_{k} f\left(x^{\prime}, u^{\prime}, 0\right) e^{i \lambda \mathbf{a} \cdot x} e^{i \frac{\lambda}{2}\left(u \cdot x^{\prime}-x \cdot u^{\prime}\right)} p_{t}^{\lambda}\left(x-x^{\prime}, u-u^{\prime}\right) d x^{\prime} d u^{\prime} \\
&=\sum_{\mathbf{m} \in \mathbb{Z}^{n}} \int_{\mathbb{R}^{2 n}} f\left(u^{\prime}+\mathbf{m}\right) e^{i \lambda x^{\prime} \cdot\left(\mathbf{m}+\frac{1}{2}\left(u+u^{\prime}+2 \mathbf{a}\right)\right)} \\
& \quad \times e^{-i \frac{\lambda}{2} x \cdot u^{\prime}} p_{t}^{\lambda}\left(x-x^{\prime}, u-u^{\prime}\right) d x^{\prime} d u^{\prime} .
\end{aligned}
$$

Using the explicit formula for $p_{t}^{\lambda}\left(x-x^{\prime}, u-u^{\prime}\right)$ the integral with respect to $d x^{\prime}$ can be seen to be

$$
\begin{aligned}
\int_{\mathbb{R}^{2 n}} e^{i \lambda x^{\prime} \cdot\left(\mathbf{m}+\frac{1}{2}\left(u+u^{\prime}+2 \mathbf{a}\right)\right)} e^{-\frac{\lambda}{4} \operatorname{coth}(\lambda t)\left(x-x^{\prime}\right)^{2}} d x^{\prime} \\
=e^{i \lambda x \cdot\left(\mathbf{m}+\frac{1}{2}\left(u+u^{\prime}+2 \mathbf{a}\right)\right)} \int_{\mathbb{R}^{2 n}} e^{-i \lambda x^{\prime} \cdot\left(\mathbf{m}+\frac{1}{2}\left(u+u^{\prime}+2 \mathbf{a}\right)\right)} e^{-\frac{\lambda}{4} \operatorname{coth}(\lambda t) x^{\prime 2}} d x^{\prime} \\
=c_{\lambda}(\tanh (\lambda t))^{\frac{n}{2}} e^{i \lambda x \cdot\left(\mathbf{m}+\frac{1}{2}\left(u+u^{\prime}+2 \mathbf{a}\right)\right)} e^{-\lambda \tanh (\lambda t)\left(\mathbf{m}+\frac{1}{2}\left(u+u^{\prime}+2 \mathbf{a}\right)\right)^{2}} .
\end{aligned}
$$

Therefore,

$$
\begin{aligned}
\int_{\mathbb{R}^{2 n}} f\left(u^{\prime}+\mathbf{m}\right) e^{i \lambda x^{\prime} \cdot\left(\mathbf{m}+\frac{1}{2}\left(u+u^{\prime}+2 \mathbf{a}\right)\right)} e^{-i \frac{\lambda}{2} x \cdot u^{\prime}} p_{t}^{\lambda}\left(x-x^{\prime}, u-u^{\prime}\right) d x^{\prime} d u^{\prime} \\
=c_{\lambda}(\sinh (2 \lambda t))^{-\frac{n}{2}} \int_{\mathbb{R}^{2 n}} f\left(u^{\prime}+\mathbf{m}\right) e^{i \lambda x \cdot\left(\mathbf{m}+\frac{1}{2}\left(u+u^{\prime}+2 \mathbf{a}\right)\right)} e^{-i \frac{\lambda}{2} x \cdot u^{\prime}} \\
\times e^{-\lambda \tanh (\lambda t)\left(\mathbf{m}+\frac{1}{2}\left(u+u^{\prime}+2 \mathbf{a}\right)\right)^{2}} e^{-\frac{\lambda}{4} \operatorname{coth}(\lambda t)\left(u-u^{\prime}\right)^{2}} d u^{\prime} .
\end{aligned}
$$

We change variables $u^{\prime} \rightarrow u^{\prime}-\mathbf{a}-\mathbf{m}$, use the expression for $K_{t}^{\lambda}$ given in (4.9) and the integral above becomes

$$
\begin{aligned}
c_{\lambda} e^{i \lambda x \cdot \mathbf{m}} e^{i \lambda x \cdot \mathbf{a}} e^{i \frac{\lambda}{2} x \cdot u} \int_{\mathbb{R}^{n}} f & \left(u^{\prime}-\mathbf{a}\right) K_{t}^{\lambda}\left(u+\mathbf{a}+\mathbf{m}, u^{\prime}\right) d u^{\prime} \\
& =c_{\lambda} e^{i \lambda x \cdot \mathbf{m}} e^{i \lambda x \cdot \mathbf{a}} e^{i \frac{\lambda}{2} x \cdot u}\left(e^{-t H(\lambda)} \tau_{\mathbf{a}} f\right)(u+\mathbf{a}+\mathbf{m}) .
\end{aligned}
$$

This completes the proof of the proposition.

We are ready for the main result in this article.

Theorem 4.7 The map

$$
T_{t}: \mathcal{H}_{k} \rightarrow \mathcal{B}_{t, \Gamma}^{\lambda}, \quad F \mapsto f *_{\lambda} p_{t}^{\lambda}
$$

is an isometric isomorphism. 
To prove the Theorem we will establish the following slightly more precise result.

Theorem 4.8 An entire function $F(z, w)$ belongs to $\mathcal{B}_{t, \mathbf{j}}^{4 \pi k}$ if and only if $F(z, w)=e^{t(4 \pi k)^{2}}\left(V_{k, \mathbf{j}} f\right) * k_{t}(z, w, 0)$ for some $f \in L^{2}\left(\mathbb{R}^{n}\right)$.

Proof. First note that the map is isometric by Proposition 4.4 and Corollary 4.3. It remains to verify surjectivity.

For that let $F \in \mathcal{B}_{t, \mathbf{j}}^{\lambda}$ with $\lambda=4 \pi k$. We have to show that there exists $f \in L^{2}\left(\mathbb{R}^{n}\right)$ such that $V_{k, \mathbf{j}} f * k_{t}(z, w, 0)=e^{-t \lambda^{2}} F(z, w)$. To prove this we consider the function

$$
G(z, w)=e^{-i \lambda \mathbf{a} \cdot z} e^{-i \frac{\lambda}{2} z \cdot w} F(z, w) .
$$

In view of the transformation properties of $F$, the function $G$ becomes $\frac{1}{2 k}$-periodic in the $x$-variables. Therefore, it admits an expansion of the form

$$
G(z, w)=\sum_{\mathbf{m} \in \mathbb{Z}^{n}} C_{\mathbf{m}}(w) e^{i \lambda \mathbf{m} \cdot z}
$$

where $C_{\mathbf{m}}$ are the Fourier coefficients:

$$
C_{\mathbf{m}}(w)=\int_{\left[0, \frac{1}{2 k}\right)^{n}} G(x, w) e^{-i \lambda \mathbf{m} \cdot x} d x .
$$

The transformation properties of $F$ lead to $G(x, w-\mathbf{m})=G(x, w) e^{i \lambda \mathbf{m} \cdot x}$ and hence $C_{\mathbf{m}}(w-\mathbf{m})=C_{\mathbf{0}}(w)$. Thus, we obtain

$$
F(z, w)=e^{i \lambda \mathbf{a} \cdot z} e^{i \frac{\lambda}{2} z \cdot w} \sum_{\mathbf{m} \in \mathbb{Z}^{n}} C_{\mathbf{0}}(w+\mathbf{m}) e^{i \lambda \mathbf{m} \cdot z} .
$$

We now show that $C_{\mathbf{0}}$ belongs to the Hermite-Bergman space $\mathcal{H}_{t}^{\lambda}$. For that we consider the finite integral:

$$
\begin{aligned}
\|F\|^{2}= & \int_{\mathbb{C}^{2 n} / \mathbb{Z}^{2 n}}|F(z, w)|^{2} W_{t}^{-\lambda}(z, w) d x d u d y d v \\
= & \int_{\mathbb{C}^{2 n} / \mathbb{Z}^{2 n}}\left|e^{2 i \lambda \mathbf{a} \cdot z} e^{i \lambda z \cdot w}\right| \cdot\left|\sum_{\mathbf{m} \in \mathbb{Z}^{n}} C_{\mathbf{0}}(w+\mathbf{m}) e^{i \lambda \mathbf{m} \cdot z}\right|^{2} \\
& \times e^{-\lambda(u \cdot y-v \cdot x)} p_{2 t}^{\lambda}(2 y, 2 v) d x d u d y d v \\
= & \sum_{\mathbf{m} \in \mathbb{Z}^{n}} \int_{\mathbb{C}^{n} / \mathbb{Z}^{n}} \int_{\mathbb{R}^{n}} e^{-2 \lambda y \cdot(a+u+\mathbf{m})}\left|C_{\mathbf{0}}(w+\mathbf{m})\right|^{2} p_{2 t}^{\lambda}(2 y, 2 v) d u d y d v
\end{aligned}
$$

We recall the explicit formula for $p_{t}^{\lambda}$ and use the fact

$$
\int_{\mathbb{R}^{n}} e^{-2 \lambda y \cdot u} e^{-\lambda \operatorname{coth}(2 \lambda t) y^{2}} d y=c_{\lambda}(\tanh (2 \lambda t))^{\frac{n}{2}} e^{\lambda \tanh (2 \lambda t) u^{2}} .
$$


As a result

$$
\|F\|^{2}=\int_{\mathbb{R}^{2 n}}\left|C_{\mathbf{0}}(w-\mathbf{a})\right|^{2} U_{t}^{\lambda}(u, v) d u d v<\infty .
$$

In view of Theorem 4.5, there exists $g \in L^{2}\left(\mathbb{R}^{n}\right)$ such that $C_{\mathbf{0}}(w)=e^{-t H(\lambda)}$ $g(w+\mathbf{a})$. Let $f=\tau_{-\mathbf{a}} g$. Then Proposition 4.6 implies

$$
F(z, w)=e^{t \lambda^{2}} V_{k, \mathbf{j}} f * k_{t}(z, w, 0)
$$

and this proves the theorem.

Remark 4.9 Our proof of the fact that the map $\left.T_{t}\right|_{\mathcal{H}_{k}}$ is isometric (Proposition 4.4) is rather robust and generalizes to all discrete subgroups $\Gamma<\mathbb{H}$. However this is not the case for our argument for the onto-ness.

Remark 4.10 Recall the finite group $\mathbf{F}_{k}$ and their two representations $\Pi_{k}$ and $\tilde{\Pi}_{k}$. Then $\left.T_{t}\right|_{\mathcal{H}_{k}}$ intertwines $\Pi_{k}$ and $\tilde{\Pi}_{k}$.

Remark 4.11 The Weil-Brezin transforms $V_{k, \mathbf{j}}$ defined on $L^{2}\left(\mathbb{R}^{n}\right)$ has a natural extension to the Hermite-Bergman spaces $\mathcal{H}_{t}^{\lambda}, \lambda=4 \pi k$. Indeed, consider the operator $\tilde{V}_{k, \mathbf{j}}$ defined on $\mathcal{H}_{t}^{\lambda}$ as follows. For $F \in \mathcal{H}_{t}^{\lambda}$ we let

$$
\tilde{V}_{k, \mathbf{j}} F(z, w, \zeta)=e^{i \lambda \zeta} e^{i \lambda \mathbf{a} \cdot z} e^{i \frac{\lambda}{2} z \cdot w} \sum_{\mathbf{m} \in \mathbb{Z}^{n}} e^{i \lambda z \cdot \mathbf{m}} F(w+\mathbf{m}) .
$$

Let us verify that the above series converges so that $\tilde{V}_{k, \mathbf{j}} F$ is well defined. As $F \in \mathcal{H}_{t}^{\lambda}$ we have

$$
F(z)=\int_{\mathbb{C}^{n}} F(w) K_{t}^{\lambda}(z, \bar{w}) U_{t}^{\lambda}(z) d z
$$

since $K_{t}^{\lambda}(z, \bar{w})$ is the reproducing kernel. From the above we get the estimate

$$
|F(z)| \leq C K_{t}^{\lambda}(z, \bar{z}) \leq C e^{-\lambda \tanh (2 \lambda t) x^{2}} e^{\lambda \operatorname{coth}(2 \lambda t) y^{2}} .
$$

Therefore, the series defining $\tilde{V}_{k, \mathbf{j}} F(z, w, \zeta)$ converges uniformly over compact subsets and defines an entire function. Moreover, we can check that $\tilde{V}_{k, \mathbf{j}} F(z, w, 0)$ belongs to $\mathcal{B}_{t, \mathbf{j}}^{\lambda}$. Thus $\tilde{V}_{k, \mathbf{j}}$ intertwines between the heat kernel transform associated to the Hermite operator and the heat kernel transform on the nilmanifold. More precisely, we have the following commutative diagram:

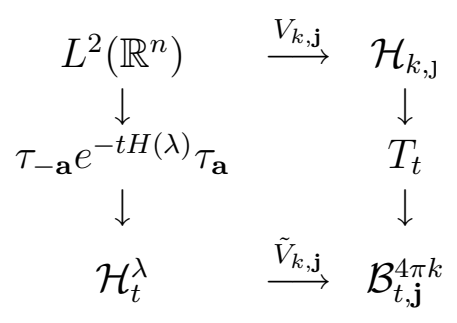


Let $\mathcal{B}_{t, \Gamma}^{0}$ be the Bergman space described in Theorem 4.1. Combining Theorems 4.1 and 4.7 we obtain the following.

Theorem 4.12 The image of $L^{2}(\Gamma \backslash \mathbb{H})$ under $T_{t}$ is the direct sum of all $\mathcal{B}_{t, \Gamma}^{4 \pi k}, k \in \mathbb{Z}$. More precisely,

$$
T_{t}\left(L^{2}(\Gamma \backslash \mathbb{H})\right)=\sum_{k=-\infty}^{\infty} e^{2 t(4 \pi k)^{2}} \mathcal{B}_{t, \Gamma}^{4 \pi k}
$$

This is the analogue of Theorem 5.1 in [8] for the heat kernel transform on the full Heisenberg group. As in the case of the full Heisenberg group, the image can be written as a direct sum of three weighted Bergman spaces if we allow the weight functions to take both positive and negative values.

Let $L_{+}^{2}(\Gamma \backslash \mathbb{H})=\oplus_{k=1}^{\infty} \mathcal{H}_{-k}$ and $L_{-}^{2}(\Gamma \backslash \mathbb{H})=\oplus_{k=1}^{\infty} \mathcal{H}_{k}$. Similarly define $L_{+}^{2}(\mathbb{T})$ and $L_{-}^{2}(\mathbb{T})$ where $\mathbb{T}=\mathbb{R} /\left(\frac{1}{2} \mathbb{Z}\right)$ is the one dimensional torus. We let $\mathcal{B}_{t}^{+}(\mathbb{C})\left(\right.$ resp. $\left.\mathcal{B}_{t}^{-}(\mathbb{C})\right)$ stand for the image of $L_{+}^{2}(\mathbb{T})\left(\right.$ resp. $\left.L_{-}^{2}(\mathbb{T})\right)$ under the heat kernel transform associated to the Laplacian on $\mathbb{T}$. These are weighted Bergman spaces that correspond to the weight $e^{-\frac{1}{2 t} y^{2}}$ which are $1 / 2$ periodic in the $x$ - variable. We define $\mathcal{B}_{t}^{+}\left(\Gamma \backslash \mathbb{H}_{\mathbb{C}}\right)$ and $\mathcal{B}_{t}^{-}\left(\Gamma \backslash \mathbb{H}_{\mathbb{C}}\right)$ as follows. Let $W_{t}^{+}$ and $W_{t}^{-}$be the weight functions that appeared in [7]. They are characterised by the conditions

$$
\int_{\mathbb{R}} W_{t}^{+}(z, w, i \eta) e^{2 \lambda \eta} d \eta=e^{2 t \lambda^{2}} W_{t}^{\lambda}(z, w)
$$

for all $\lambda>0$ and

$$
\int_{\mathbb{R}} W_{t}^{-}(z, w, i \eta) e^{2 \lambda \eta} d \eta=e^{2 t \lambda^{2}} W_{t}^{\lambda}(z, w)
$$

for all $\lambda<0$.

We consider an exhaustion of $\Gamma \backslash \mathbb{H}$ defined as follows. For each positive integer $m$ let $E_{m}=\left\{z=x+i y \in \mathbb{C}^{n}: x \in[0,1)^{n},|y| \leq m\right\}$ and $E=$ $[0,1) \times \mathbb{R}$. We define $K_{m}=E_{m} \times E_{m} \times E$ so that the union of all $K_{m}$ as $m$ varies over all positive integers is just $\Gamma \backslash \mathbb{H}$. We let $\mathcal{V}^{+}\left(\Gamma \backslash \mathbb{H}_{\mathbb{C}}\right)$ stand for the space all entire functions $F$ on $\mathbb{C}^{n} \times \mathbb{C}^{n} \times \mathbb{C}$ such that $F \in L^{2}\left(K_{m},\left|W_{t}^{+}\right| d g\right)$ for all $m$;

$$
\lim _{m \rightarrow \infty} \int_{K_{m}}|F(g)|^{2} W_{t}^{+}(g) d g<\infty
$$

and $F(z, w, \cdot) \in \mathcal{B}_{t}^{+}(\mathbb{C})$ for all $z, w \in \mathbb{C}^{n}$. We equip $\mathcal{V}^{+}\left(\Gamma \backslash \mathbb{H}_{\mathbb{C}}\right)$ with the sesquilinear product

$$
(F, G)_{+}=\lim _{m \rightarrow \infty} \int_{K_{m}} F(g) \bar{G}(g) W_{t}^{+}(g) d g .
$$


We similarly define $\mathcal{V}^{-}\left(\Gamma \backslash \mathbb{H}_{\mathbb{C}}\right)$. As in [8, Lemma 6.4], one shows that the above bracket is positive definite and we define $\mathcal{B}_{t}^{+}\left(\Gamma \backslash \mathbb{H}_{\mathbb{C}}\right)$ and $\mathcal{B}_{t}^{-}\left(\Gamma \backslash \mathbb{H}_{\mathbb{C}}\right)$ as the completions of $\mathcal{V}^{+}\left(\Gamma \backslash \mathbb{H}_{\mathbb{C}}\right)$ and $\mathcal{V}^{-}\left(\Gamma \backslash \mathbb{H}_{\mathbb{C}}\right)$. We also define $\mathcal{B}_{t}^{0}\left(\Gamma \backslash \mathbb{H}_{\mathbb{C}}\right)$ to be the space described in Theorem 4.1. With these definitions we have the following result.

\section{Theorem 4.13}

$$
T_{t}\left(L^{2}(\Gamma \backslash \mathbb{H})\right)=\mathcal{B}_{t}^{-}\left(\Gamma \backslash \mathbb{H}_{\mathbb{C}}\right) \oplus \mathcal{B}_{t}^{0}\left(\Gamma \backslash \mathbb{H}_{\mathbb{C}}\right) \oplus \mathcal{B}_{t}^{+}\left(\Gamma \backslash \mathbb{H}_{\mathbb{C}}\right)
$$

Proof. The proof is similar to the case of the full Heisenberg group. So we will be very sketchy. We refer to [7] for details (see Lemma 6.4 and Theorem 6.4). First we check that the above sesquilinear product defines a pre-Hilbert space structure. The condition $F(z, w, \cdot) \in \mathcal{B}_{t}^{+}(\mathbb{C})$ shows that there exists a function $G(z, w, \cdot) \in L_{+}^{2}(\mathbb{T})$ such that

$$
F(z, w, \zeta)=\int_{\mathbb{R}} G(z, w, s) q_{t}(\zeta-s) d s
$$

where $q_{t}$ is the Euclidean heat kernel. Therefore, up to a multiplicative constant

$$
\int_{[0,1 / 2)} F(z, w, \xi+i \eta) e^{-4 \pi i k \xi} d \xi=e^{-4 \pi k \eta} e^{-t(4 \pi k)^{2}} G^{-4 \pi k}(z, w) .
$$

Since $W_{t}^{+}$is independent of $\xi$ we have

$$
\begin{aligned}
& \int_{K_{m}}|F(g)|^{2} W_{t}^{+}(g) d g \\
& =\int_{E_{m} \times E_{m}} \int_{\mathbb{R}} \sum_{k=-\infty}^{-1}\left|G^{-4 \pi k}(z, w)\right|^{2} e^{-2(4 \pi k) \eta} e^{-2 t(4 \pi k)^{2}} W_{t}^{+}(z, w, i \eta) d z d w d \eta .
\end{aligned}
$$

In view of the defining property of $W_{t}^{+}$the above reduces to

$$
\int_{E_{m} \times E_{m}} \sum_{k=-\infty}^{-1}\left|G^{-4 \pi k}(z, w)\right|^{2} W_{t}^{-4 \pi k}(z, w) d z d w .
$$

This shows that the sesquilinear form is positive definite.

Consider the subspace $L_{+, 0}^{2}(\Gamma \backslash \mathbb{H})$ consisting of functions in $L_{+}^{2}(\Gamma \backslash \mathbb{H})$ with only finitely many nonzero Fourier coefficients. Then it is dense in $L_{+}^{2}(\Gamma \backslash \mathbb{H})$. The proof will be complete if we can show that

$$
T_{t}\left(L_{+, 0}^{2}(\Gamma \backslash \mathbb{H})\right) \subset \mathcal{V}^{+}\left(\Gamma \backslash \mathbb{H}_{\mathbb{C}}\right) \subset T_{t}\left(L_{+}^{2}(\Gamma \backslash \mathbb{H})\right)
$$

and the inclusions are isometric. 
Suppose $F=f * k_{t}$ with $f \in L_{+, 0}^{2}(\Gamma \backslash \mathbb{H})$. Then $f=\sum_{j=-N}^{-1} f_{j}, f_{j} \in \mathcal{H}_{j}$ for some $N$. Since

$$
f_{j} * k_{t}(z, w, \zeta)=e^{-t(4 \pi j)^{2}} e^{4 \pi i j \zeta} g_{j} *_{-4 \pi j} p_{t}^{-4 \pi j}(z, w)
$$

where $g_{j}(x, u)=f_{j}(x, u, 0)$ a simple calculation shows that

$$
\begin{aligned}
\lim _{m \rightarrow \infty} \int_{K_{m}} \mid & \left.F(g)\right|^{2} W_{t}^{+}(g) d g \\
& =\sum_{j=-N}^{-1} \int_{[0,1)^{2 n}} \int_{\mathbb{R}^{2 n}}\left|g_{j} *_{-4 \pi j} p_{t}^{-4 \pi j}(z, w)\right|^{2} W_{t}^{-4 \pi j}(z, w) d z d w \\
& =C \sum_{j=-N}^{-1} \int_{[0,1)^{2 n}}\left|g_{j}(x, u)\right|^{2} d x d u=C \int_{\Gamma \backslash \mathbb{H}}|f(h)|^{2} d h .
\end{aligned}
$$

This proves that $T_{t}\left(L_{+, 0}^{2}(\Gamma \backslash \mathbb{H})\right)$ is isometrically embedded into $\mathcal{V}^{+}\left(\Gamma \backslash \mathbb{H}_{\mathbb{C}}\right)$.

To prove the inclusion $\mathcal{V}^{+}\left(\Gamma \backslash \mathbb{H}_{\mathbb{C}}\right) \subset T_{t}\left(L_{+}^{2}(\Gamma \backslash \mathbb{H})\right)$ let us take $F \in \mathcal{V}^{+}\left(\Gamma \backslash \mathbb{H}_{\mathbb{C}}\right)$ and consider the representation

$$
F(z, w, \zeta)=\int_{\mathbb{R}} G(z, w, s) q_{t}(\zeta-s) d s
$$

The argument used to prove the positive definiteness of the inner product on $\mathcal{V}^{+}\left(\Gamma \backslash \mathbb{H}_{\mathbb{C}}\right)$ shows that $G^{-4 \pi k}(z, w) \in \mathcal{B}_{t, \Gamma}^{-4 \pi k}$ for $-\infty<k \leq-1$. We can write $G^{-4 \pi k}(z, w)=g^{-4 \pi k} *_{-4 \pi k} p_{t}^{-4 \pi k}(z, w)$ for some functions $g^{-4 \pi k}$. If we define

$$
f(x, u, \xi)=\sum_{-\infty}^{-1} e^{4 \pi i k \xi} g^{-4 \pi k}(x, u)
$$

then it follows that $f * k_{t}(z, w, \zeta)=F(z, w, \zeta)$ as desired. This completes the proof.

\section{References}

11] Auslander, L. And Brezin, J.: Translation invariant subspaces in $L^{2}$ of a compact nilmanifold. I. Invent. Math. 20 (1973), 1-14.

[2] Bargmann, V.: On a Hilbert space of analytic functions and an associated integral transform. Comm. Pure Appl. Math. 14 (1961), 187-214.

[3] Brezin, J.: Harmonic analysis on nilmanifolds. Trans. Amer. Math. Soc. 150 (1970), 611-618.

[4] Byun, D.-W.: Inversions of Hermite semigroup. Proc. Amer. Math. Soc. 118 (1993), 437-445. 
[5] Folland, G. B.: Compact Heisenberg manifolds as CR manifolds. J. Geom. Anal. 14 (2004), 521-532.

[6] Gelf̌and, I., Graev, M. and Pyatetskit-Shapiro, I.: Representation theory and automorphic functions. Generalized Functions 6. Academic Press, Boston, MA, 1990.

[7] Hall, B.: The Segal-Bargmann "coherent state" transform for compact Lie groups. J. Funct. Anal. 122 (1994), no. 1, 103-151.

[8] Krötz, B., Thangavelu, S. And Xu, Y.: The heat kernel transform for the Heisenberg group. J. Funct. Anal. 225 (2005), no. 2, 301-336.

[9] Krötz, B., Olafsson, G. and Stanton, R.: The image of the heat kernel transform on Riemannian symmetric spaces of noncompact type. Int. Math. Res. Not. 2005, no. 22, 1307-1329.

[10] Raghunathan, M.S.: Discrete subgroups of Lie groups. Ergebnisse der Mathematik und ihrer Grenzgebiete 68. Springer-Verlag, New YorkHeidelberg, 1972.

[11] Stenzel, M.: The Segal-Bargmann transform on a symmetric space of compact type. J. Funct. Anal. 165 (1999), no. 1, 44-58.

[12] Thangavelu, S.: Harmonic analysis on the Heisenberg group. Progress in Mathematics 159. Birkhäuser Boston, Boston, MA, 1998.

[13] Tolimieri, R.: The theta transform and the Heisenberg group. J. Funct. Anal. 24 (1977), 353-363.

Recibido: 6 de julio de 2006

Revisado: 14 de noviembre de 2006

Bernhard Krötz

Max-Planck Institut für Mathematik Vivatsgasse 7, D-53111 Bonn, Germany kroetz@mpim-bonn.mpg.de

Sundaram Thangavelu Department of Mathematics Indian Institute of Science

Bangalore 560 012, India veluma@math.iisc.ernet.in

Yuan Xu

Department of Mathematics University of Oregon Eugene, OR 97403, USA yuan@math . oregon . edu 